\title{
Cryo-electron Tomography Analysis of Infectious Extracellular Vesicles from a Non-enveloped RNA Virus
}

\author{
Jie Yang $^{1}$, Evan Rossignol ${ }^{1}$, Esther Bullitt ${ }^{1}$ \\ ${ }^{1 .}$ Boston University School of Medicine, Department of Physiology \& Biophysics, Boston, MA
}

Extracellular vesicles (EVs) comprise a wide variety of vesicles shed from cells into the extracellular space. EVs carry and transport proteins, lipids, RNA (mRNA, microRNA, noncoding sequences), and DNA. The abundant presence of EVs in body fluids such as blood, urine and saliva, suggests its role in enabling local and distal cell-to-cell communication ([1] and reviewed in [2,3]). Extensive studies have shown that in enveloped virus-infected cells, such as Human immunodeficiency virus (HIV), viral infection modulates EVs' sorting and release, enabling transport of infectious viral genomic sequences (reviewed in [4]). In addition, EVs also manifest themselves as an unconventional intercellular transport mechanism for non-enveloped, positive-sense single-stranded RNA viruses, such as Poliovirus and Coxsackievirus [5]. Prior to cell lysis, phosphatidylserine (PS)-rich EVs can simultaneously transport multiple viruses to propagate infection [5]. However little is known about these infectious extracellular vesicles (IEVs).

Major cellular pathways require spatial organization of the essential biological molecules. Threedimensional (3D) structural analysis is fundamental in understanding these pathways. Researchers have previously reported on the morphology of IEVs through conventionally prepared samples, visualized using transmission electron microscopy (TEM) [5,6]. Despite advances in our knowledge gained from these data, potential artifacts limit understanding of IEVs. Here, we present original results on highresolution reconstructed 3D structures of fully hydrated, unstained native IEVs.

IEVs from poliovirus-infected cells, a prototype for non-enveloped positive-sense single-stranded RNA viruses, were purified, fixed, and applied to holey carbon-coated grids. An additional layer of_carbon was added in our lab, retaining the holes and stabilizing the carbon, to better preserve the samples. IEVs were plunge-frozen and imaged at $160 \mathrm{kV}$. tilt series' were recorded from -60 to +60 degree, and data were aligned and reconstructed using IMOD software [7]. These cryo-electron tomography (cryoET) data have allowed us to reconstruct the first 3D structures of native IEVs. Our cryoET data show that IEVs $(\mathrm{n}=177)$ are single-membrane bounded, with a size range of 52 to $822 \mathrm{~nm}$ in diameter, with over $70 \%$ of the IEV diameters 100 to $300 \mathrm{~nm}$. As shown in Figure 1, clusters of 10 to 30 virions $\sim 28 \mathrm{~nm}$ diameter, with a resolution of $5.7 \mathrm{~nm}(\mathrm{n}=509)$, are visible within the IEVs. The virions are either densely packed, in a well-ordered arrangement within IEVs (Panel A and D), or are concentrated at one end of the IEV, forming a polar IEV structure (Panel G). In addition to virions, IEVs often contain internal vesicles 60-100 nm in diameter; "hair-ball-like" structures with a strong, tangled-looking, thread-like electron density; and/or partially assembled virion-like structures. Occasionally, we observed larger IEVs with multiple internal vesicular structures and close to 100 densely packed virions. Head-stem-like membrane-associated structures were observed within some internal vesicles of the IEVs. From these data we hypothesize that in addition to clusters of virions, IEVs carry and transport other biological components.

IEVs represent a novel interaction mechanism between the host and pathogen, potentially shedding light on understanding latent viral infection by viruses [8] that remains problematic. In future work, we will 
study IEVs' structural features through a combined approach that includes advanced Single Particle Interferometric Reflectance Imaging Sensor (SP-IRIS) [9] and cryo-ET. Additional future research will focus on identifying the IEV-transported components and elucidating the mechanism underlying the formation of IEVs in cells infected by non-enveloped viruses [10].

\section{References}

[1] ML Squadrito et al. Cell Rep. 2014, 8, p. 1432-1446.

[2] HJ Huber, P Holvoet. Curr. Opin. Lipidol. 2015, 26, p. 412-419.

[3] JS Schorey et al. EMBO Rep. 2015, 16, p. 24-43.

[4] MN Madison, CM Okeoma. Viruses 2015, 7, p. 4093-4118.

[5] YH Chen et al. Cell 2015, 160, p. 619-630.

[6] SM Robinson et al. PLoS Pathog. 2014, 10, p. e1004045.

[7] DN Mastronarde, SR Held. J. Struct. Biol. 2017, 197, p. 102-113.

[8] SR Baglio et al. Proc. Natl. Acad. Sci. 2016, 113, p. E587-E596.

[9] GG Daaboul, et al. Sci. Rep. 2016, 6, p. 37246.

[10] This research was supported by the NIH Grant (GM102474 to Esther Bullitt).
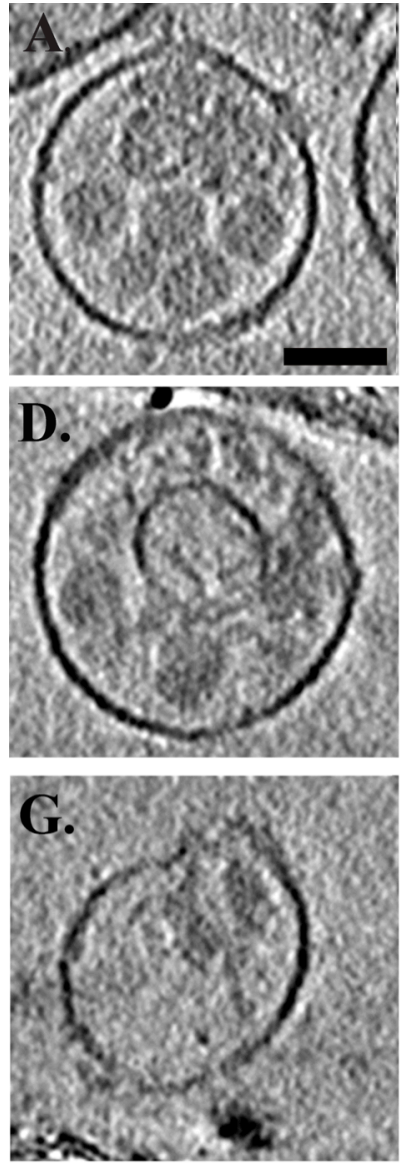
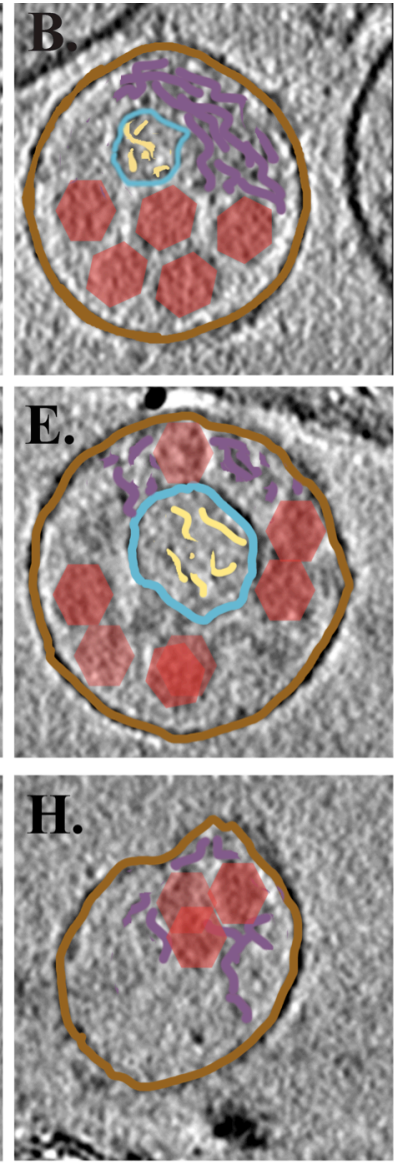
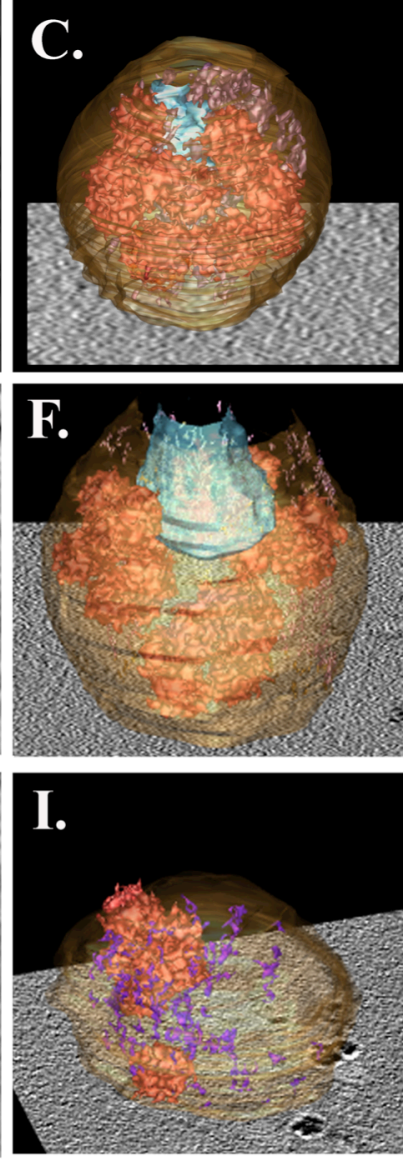

Figure 1. Isolated extra-cellular vesicles from virus-infected cells revealed by cryo-ET. 3D reconstructed structures of cryopreserved IEVs are shown in tomographic slices $82 \mathrm{~nm}$ thick in Panels (A), (D). These slices each display a single membrane vesicle bounding multiple viruses (modeled as red hexagons in B, E), a capsid-like structure with low inner density (B), a vesicular structure (E) (modeled in blue), and some hairball-like substances with electron density traced in purple in (B) and (E). Panels (C) and (F) show 3D surface rendering of the same vesicles shown in (A) and (D), respectively, rotated around the $\mathrm{x}$-axis by $65^{\circ}$. A-F. Clusters of 15-40 virions (red), "incomplete" virions or vesicles (blue) and

purple-colored hairball-like substances are carried in a singled-membraned, irregularly-shaped vesicle (brown). Panel (G) presents another class of IEV where virions (red) are arranged at one end of the vesicle, surrounded by density (purple), similar to that observed in panels A and B. (I) shows a 3D surface rendering of the tomogram, rotated $65^{\circ}$ about the $\mathrm{x}$-axis from the image in panel G. For all images in Figure 1, the scale bar represents $50 \mathrm{~nm}$; images are magnified 3x from the primary data. 